\title{
Radiance Caching and Local Geometry Correction
}

\author{
Okan Arikan \\ David A. Forsyth \\ James F. O'Brien
}

University of California, Berkeley

\begin{abstract}
We present a final gather algorithm which splits the irradiance integral into two components. One component captures the incident radiance due to distant surfaces. This incident radiance due to far field illumination is represented as a spatially varying field of spherical harmonic coefficients. Since distant surfaces do not cause rapid changes in incident radiance, this field is smooth and slowly varying and can be computed quickly and represented efficiently.

In contrast, nearby surfaces may create drastic changes in irradiance, because their positions on the visible hemisphere can change quickly. We can find such nearby surfaces (scene triangles) by a local search. By assuming nearby surfaces are always visible, we can correct the far field irradiance estimate we obtain using the spherical harmonics, and restore the high frequency detail in indirect lighting. This correction can be performed efficiently because finding nearby surfaces is a local operation.
\end{abstract}

\section{Method}

We describe an approximation method for final gathering that achieves about an order of magnitude speedup over traditional irradiance caching based methods, without noticeable error on a large variety of scenes.

The irradiance integral represents the illumination that a point receives from all other visible surfaces. Some of these visible surfaces may be nearby (for our purposes, closer than a threshold $\alpha$ ) and some may be far away (farther away than $\alpha$ ). Indirect illumination coming from distant surfaces does not change very rapidly as one moves about on the receiver. This means that it can be represented with a very sparse set of samples [Ward et al. 1988]. As we move on a surface, nearby surfaces move significantly on the incident hemisphere of the point we're interested in. This means irradiance cache based approaches must collect samples densely around the high surface detail in order to capture the potentially rapidly varying term due to nearby surfaces. We take advantage of this by separating the incident radiance at a point into two components: far field radiance from distant surfaces and near field radiance from nearby surfaces.

Incident radiance from distant surfaces can be represented using only low order spherical harmonics [Ramamoorthi and Hanrahan 2001]. Because the source of the radiance is distant, the coefficients of the harmonics don't change very rapidly on the surfaces. This allows us to compute the incident radiance representation as a set of harmonic coefficients at a sparse set of locations and interpolate. Due to the properties of spherical harmonics, the irradiance integral over the incident directions can be evaluated efficiently as a dot product of harmonic coefficients.

The effect of the nearby surfaces can be determined explicitly. In our implementation, a scene is described as a set of triangles. Given a shading point, nearby triangles can be found efficiently using space partitioning algorithms. Because nearby triangles are usually visible (they are nearby), we can obtain an approximation to the energy they reflect or occlude by ignoring the visibility term in the form factor integral. This local energy correction term can be efficiently computed using a analytical point-to-polygon form factors. It is this correction term which restores the high frequency detail

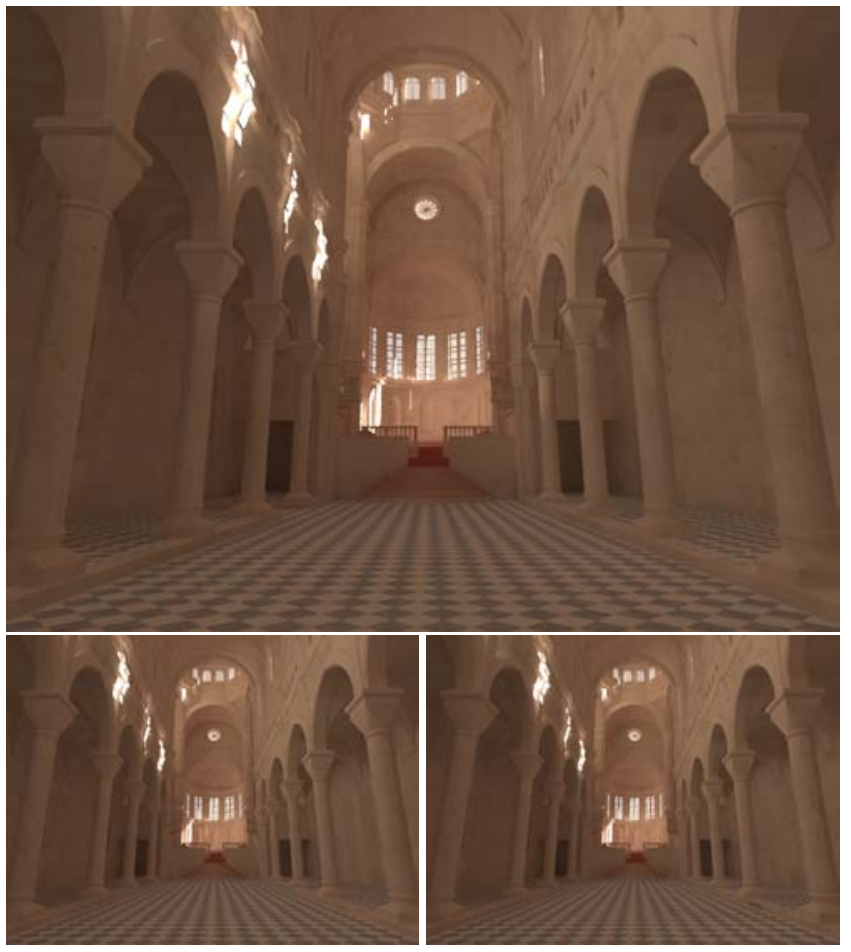

Figure 1: The top image demonstrates our final gather algorithm. This scene is illuminated with a single distant light source and most of the lighting is indirect. The top image is computed in $5 \mathrm{~min}-$ utes on an Athlon 2.2. The bottom right image is obtained using irradiance caching in 1 hour. The bottom-left image shows the ground truth obtained using Monte Carlo techniques. Notice that our method is over an order of magnitude faster than irradiance caching and is very close to the ground truth in terms of quality.

in the indirect illumination which is crucial for realism and also traditionally is very expensive to compute using irradiance caching based approaches.

Figure 1 shows a result of our algorithm and compares it to ground truth and the results from irradiance caches. In terms of quality, our results compare well to the ground truth. We are able to account for high frequency change in the indirect illumination, which has been traditionally very expensive. We further demonstrate in the supplemental figures, that we obtain an order of magnitude speedup on a variety of complicated scenes that have high surface detail.

\section{References}

RAmamoorthi, R., AND HANRAhan, P. 2001. The relationship between radiance and irradiance: Determining the illumination from images of a convex lambertian object. In Journal of the Optical Society of America.

WARD, G., RUBinsteIn, F., AND ClEAR, R. 1988. A ray tracing solution for diffuse interreflectio. In SIGGRAPH 1988, Computer Graphics Proceedings. 